\section{CRYSTALLOGRAPHIC 듣 COMMUNICATIONS}

ISSN 2056-9890

Received 20 January 2015

Accepted 16 March 2015

Edited by M. Weil, Vienna University of Technology, Austria

Keywords: crystal structure; ruthenocene ruthenocenecarbonitrile; sandwich compound; nitrile

CCDC reference: 1054219

Supporting information: this article has supporting information at journals.iucr.org/e

\section{Crystal structure of ruthenocenecarbonitrile}

\author{
Frank Strehler, Marcus Korb and Heinrich Lang*
}

Technische Universität Chemnitz, Fakultät für Naturwissenschaften, Institut für Chemie, Anorganische Chemie, D-09107 Chemnitz, Germany. *Correspondence e-mail: heinrich.lang@chemie.tu-chemnitz.de

The molecular structure of ruthenocenecarbonitrile, $\left[\mathrm{Ru}\left(\eta^{5}-\mathrm{C}_{5} \mathrm{H}_{4} \mathrm{C} \equiv \mathrm{N}\right)\left(\eta^{5}\right.\right.$ $\left.\mathrm{C}_{5} \mathrm{H}_{5}\right)$ ], exhibits point group symmetry $m$, with the mirror plane bisecting the molecule through the $\mathrm{C} \equiv \mathrm{N}$ substituent. The $\mathrm{Ru}^{\mathrm{II}}$ atom is slightly shifted from the $\eta^{5}-\mathrm{C}_{5} \mathrm{H}_{4}$ centroid towards the $\mathrm{C} \equiv \mathrm{N}$ substituent. In the crystal, molecules are arranged in columns parallel to [100]. One-dimensional intermolecular $\pi-\pi$ interactions $[3.363$ (3) $\AA]$ between the $\mathrm{C} \equiv \mathrm{N}$ carbon atom and one carbon of the cyclopentadienyl ring of the overlaying molecule are present.

\section{Chemical context}

The nitrile group is isoelectronic with the acetylid function (Bonniard et al., 2011), which has already been investigated in electron-transfer studies (see, for example, Lang et al., 2006; Poppitz et al., 2014; Speck et al., 2012; Hildebrandt \& Lang, 2013; Miesel et al., 2013). Coordination of, for example, ferrocenecarbonitrile towards transition metals $M$ will allow investigation of the electronic properties of $-\mathrm{C} \equiv \mathrm{N}-M-$ or $-\mathrm{C} \equiv \mathrm{N}-M-\mathrm{N} \equiv \mathrm{C}-$ bridging units. A synthesis for ferrocenecarbonitrile has already been described in 1957 (Graham et al., 1957); however, only one example of an application in electrochemical studies has been described by Dowling et al. (1981). This prompted us to synthesize ferrocenecarbonitrile transition metal complexes to investigate the electronic properties of the $-\mathrm{C} \equiv \mathrm{N}-M-\mathrm{N} \equiv \mathrm{C}-$ bridging units (Strehler et al. 2013, 2014). In a continuation of this work, we present herein the synthesis and crystal structure of the related ruthenocenecarbonitrile, (I). The synthesis of this compound was realized by treatment of formylruthenocene with hydroxylamine hydrochloride, zinc oxide and potassium iodide in acetonitrile, which is similar to a procedure already described for the synthesis of ferrocenecarbonitrile (Kivrak \& Zora, 2007).

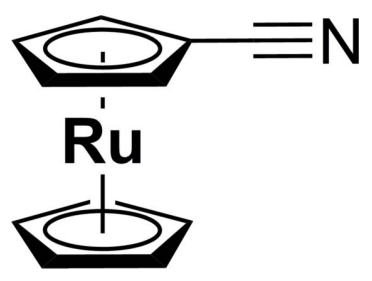

\section{Structural commentary}

The title compound contains one half-molecule in the asymmetric unit with a mirror plane bisecting the molecule through atoms $\mathrm{C} 1, \mathrm{C} 2, \mathrm{C} 5, \mathrm{~N} 1$ and Ru1 (Fig. 1). The Ru1-centroid distance to the $\mathrm{C} \equiv \mathrm{N}$-substituted cyclopentadienyl ring is 
Table 1

Selected bond lengths $(\AA)$ and angles $\left(^{\circ}\right)$ for the clarification of the shift of the Ru1 atom towards the $\mathrm{C} \equiv \mathrm{N}$ substituent in (I).

$D$ is the centroid of the $\mathrm{C}_{5} \mathrm{H}_{4}$ or $\mathrm{C}_{5} \mathrm{H}_{5}$ ring.

\begin{tabular}{lllllll}
\hline & $\mathrm{C} 2$ & $\mathrm{C} 3$ & $\mathrm{C} 4$ & $\mathrm{C} 5$ & $\mathrm{C} 6$ & $\mathrm{C}$ \\
\hline $\mathrm{Ru} 1-\mathrm{C}$ & $2.1650(18)$ & $2.1886(13)$ & $2.2013(12)$ & $2.1779(18)$ & $2.1847(13)$ & $2.1879(12)$ \\
$\mathrm{C}-D-\mathrm{Ru} 1$ & $88.90(8)$ & $89.63(6)$ & $90.93(6)$ & $89.75(9)$ & $89.95(6)$ & $90.16(6)$ \\
\hline
\end{tabular}

slightly increased [1.8179(1) $\AA$ ] compared to the unsubstituted $\mathrm{C}_{5} \mathrm{H}_{5}$ unit [1.8157 (1) $\AA$ ]. Both cyclopentadienyl rings adopt an ideally eclipsed conformation and are virtually oriented parallel towards each other, which is expressed by the bond angle at the $\mathrm{Ru}^{\mathrm{II}}$ between the two centroids $(=D)$, with $D\left(\mathrm{C}_{5} \mathrm{H}_{4}\right)-\mathrm{Ru} 1-D\left(\mathrm{C}_{5} \mathrm{H}_{5}\right)=178.87(1)^{\circ}$. However, the $\mathrm{Ru}^{\mathrm{II}}$ atom is slightly shifted from the centre of the $\mathrm{C}_{5}$ ring to the nitrile-bonded $\mathrm{C} 2$ atom, which can be explained best by the significantly different $\mathrm{Ru}-\mathrm{C}$ bond lengths (Table 1 ) and also the $\mathrm{Ru}-D-\mathrm{C}$ angles, which should ideally be $90^{\circ}$ (Table 1 ). This is in accordance with the shift in the ferrocenedicarbonitrile structure (Altmannshofer et al., 2008). The $\mathrm{C} \equiv \mathrm{N}$ substituent itself is bent away from the metal atom in (I), with a maximum shift for N1 [0.047 (4) $\AA$ ] .

\section{Supramolecular features}

The packing of (I) consists of a layer-type structure parallel to (010) with the direction of the $\mathrm{C} \equiv \mathrm{N}$ function aligned parallel to $[10 \overline{1}]$, alternating between adjacent layers. A further order is observed by a columnar arrangement of slightly tilted molecules parallel to [100]. Weak intermolecular $\pi-\pi$ interactions within the sum of the van der Waals radii $(\Sigma=3.4 \AA$; Bondi, 1964) are present between $\mathrm{C} 5$ and the $\mathrm{C} 1^{\prime}$ atom [3.363(3) $\AA]$ of the overlying molecule in the same layer (Fig. 2).

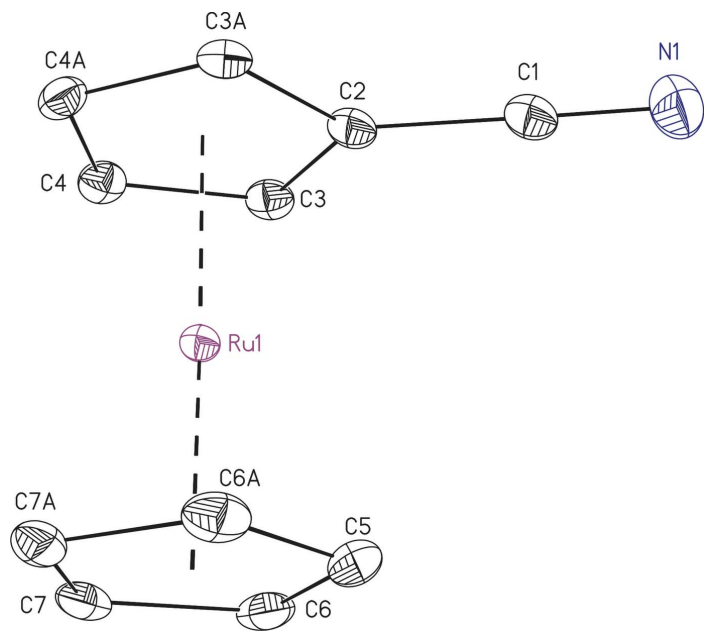

Figure 1

The molecular structure of (I), with displacement ellipsoids drawn at the $50 \%$ probability level. All $\mathrm{H}$ atoms have been omitted for clarity. [Symmetry code: (A) $x,-y+\frac{1}{2}, z$.]

\section{Database survey}

The ruthenocene backbone is hardly described in the literature. Reported derivatives contain $s p$ (ethynyl) (Sato et al., 1997; Packheiser et al., 2008; Jakob et al., 2008, 2009a), sp ${ }^{2}$ (Sato et al., 1998, 2004; Jakob et al., 2009b) and $s p^{3}$ (Sokolov et al., 2010; Barlow et al., 2001) carbon substituents or a carboxylic acid moiety (Zhang \& Coppens, 2001) and its respective $\mathrm{Ru}^{\mathrm{II}}$ complex (Wyman et al., 2005). They all exhibit similar $\mathrm{Ru}-D$ distances (1.795-1.823 $\AA$ ) as compared to (I) [1.8179 (1)-1.8157 (1) ^] or unsubstituted ruthenocene (1.794-1.816 ̊) (Ma \& Coppens, 2003; Borissova et al., 2008; Seiler \& Dunitz, 1980).

Comparison of the $\mathrm{C}-\mathrm{C}[1.431(3) \AA]$ and the $\mathrm{C} \equiv \mathrm{N}$ distances $[1.148$ (3) $\AA]$ with the respective ferrocene carbonitrile derivatives $(\mathrm{C} \equiv \mathrm{N}=1.133-1.150 ; \quad \mathrm{C}-\mathrm{C}=1.428$ 1.433 А; Altmannshofer et al., 2008; Dayaker et al., 2010; Bell et al., 1996; Nemykin et al., 2007; Erben et al., 2007) reveals no significant influence of the central metal atom on the electronic properties of the substituent.

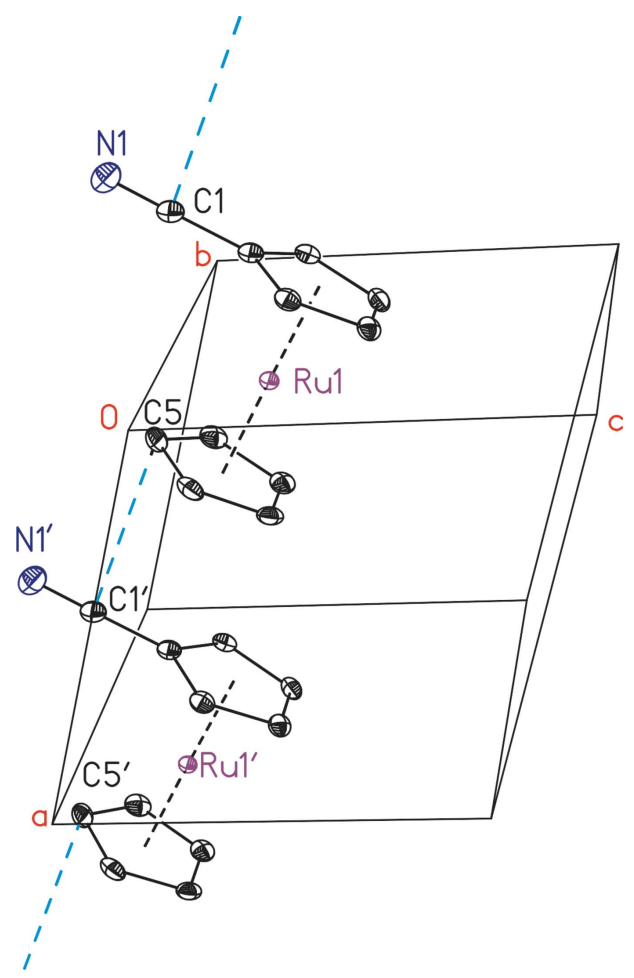

Figure 2

Intermolecular $\pi-\pi$ interactions (blue) between $\mathrm{C} 5$ and $\mathrm{C} 1^{\prime}$ in the crystal structure of (I). All $\mathrm{H}$ atoms have been omitted for clarity. [Symmetry code: $\left({ }^{\prime}\right) x-1, y, z$.] 
Table 2

Experimental details.

\begin{tabular}{|c|c|}
\hline \multicolumn{2}{|l|}{ Crystal data } \\
\hline Chemical formula & {$\left[\mathrm{Ru}\left(\mathrm{C}_{5} \mathrm{H}_{5}\right)\left(\mathrm{C}_{6} \mathrm{H}_{4} \mathrm{~N}\right)\right]$} \\
\hline$M_{\mathrm{r}}$ & 256.26 \\
\hline Crystal system, space group & Monoclinic, $P 2_{1} / m$ \\
\hline Temperature $(\mathrm{K})$ & 110 \\
\hline$a, b, c(\AA)$ & 7.2023 (2), 8.6802 (2), $7.2922(1)$ \\
\hline$\beta\left(^{\circ}\right)$ & $106.497(2)$ \\
\hline$V\left(\AA^{3}\right)$ & $437.12(2)$ \\
\hline$Z$ & 2 \\
\hline Radiation type & Мо $K \alpha$ \\
\hline$\mu\left(\mathrm{mm}^{-1}\right)$ & 1.74 \\
\hline Crystal size $(\mathrm{mm})$ & $0.38 \times 0.30 \times 0.30$ \\
\hline \multicolumn{2}{|l|}{ Data collection } \\
\hline Diffractometer & Oxford Gemini S CCD \\
\hline Absorption correction & $\begin{array}{l}\text { Multi-scan (CrysAlis RED; Oxford } \\
\text { Diffraction, 2006) }\end{array}$ \\
\hline$T_{\min }, T_{\max }$ & $0.849,1.000$ \\
\hline $\begin{array}{l}\text { No. of measured, independent and } \\
\text { observed }[I>2 \sigma(I)] \text { reflections }\end{array}$ & $27710,900,877$ \\
\hline$R_{\text {int }}$ & 0.019 \\
\hline$(\sin \theta / \lambda)_{\max }\left(\AA^{-1}\right)$ & 0.617 \\
\hline \multicolumn{2}{|l|}{ Refinement } \\
\hline$R\left[F^{2}>2 \sigma\left(F^{2}\right)\right], w R\left(F^{2}\right), S$ & $0.012,0.032,1.05$ \\
\hline No. of reflections & 900 \\
\hline No. of parameters & 67 \\
\hline $\mathrm{H}$-atom treatment & H-atom parameters constrained \\
\hline$\Delta \rho_{\max }, \Delta \rho_{\min }\left(\mathrm{e} \AA^{-3}\right)$ & $0.27,-0.39$ \\
\hline
\end{tabular}

Computer programs: CrysAlis CCD and CrysAlis RED (Oxford Diffraction, 2006), SHELXS97 and SHELXTL (Sheldrick, 2008), SHELXL2013 (Sheldrick, 2015), ORTEP-3 for Windows and WinGX (Farrugia, 2012) and publCIF (Westrip, 2010).

\section{Synthesis and crystallization}

Formylruthenocene was prepared according to a published procedure (Mueller-Westerhoff et al., 1993). Synthesis of ruthenocenecarbonitrile, (I): formylruthenocene $(2.27 \mathrm{~g}$, $8.8 \mathrm{mmol})$, hydroxylamine hydrochloride $(0.96 \mathrm{~g}, 13.8 \mathrm{mmol})$, zinc oxide $(0.86 \mathrm{~g}, 10.6 \mathrm{mmol})$ and potassium iodide $(1.76 \mathrm{~g}$, $10.6 \mathrm{mmol}$ ) were suspended in $120 \mathrm{ml}$ of dry acetonitrile. The mixture was stirred for $4 \mathrm{~h}$ at precisely $368 \mathrm{~K}$. After cooling the reaction mixture to ambient temperature, $18 \mathrm{ml}$ of an aqueous $\mathrm{Na}_{2} \mathrm{~S}_{2} \mathrm{O}_{3}(5 \%)$ solution were added in a single portion, and stirring was continued for additional $20 \mathrm{~min}$. Solid particles were removed by filtration and the filtrate was extracted with ethyl acetate $(3 \times 50 \mathrm{ml})$. The combined organic layers were dried over $\mathrm{MgSO}_{4}$. All volatiles were removed under reduced pressure and the crude product was purified by flash chromatography on aluminum oxide using dichloromethane as eluent. Greenish crystals of (I) were obtained by slow evaporation of a saturated dichloromethane solution containing (I) at ambient temperature (yield: $820 \mathrm{mg}$, $3.3 \mathrm{mmol}, 38 \%$ based on formylruthenocene). IR $\left(\mathrm{KBr}, \mathrm{cm}^{-1}\right)$ : $v=2226(m, \mathrm{C} \equiv \mathrm{N}), 2854(s), 2925(s), 3082(m, \mathrm{C}-\mathrm{H}) .{ }^{1} \mathrm{H}$ NMR (500.3 MHz, $\left.\mathrm{CDCl}_{3}, 298 \mathrm{~K}\right): \delta 4.69\left(s, 5 \mathrm{H}, \mathrm{C}_{5} \mathrm{H}_{5}\right), 4.70$ $\left(p t, 2 \mathrm{H}, J_{\mathrm{H}, \mathrm{H}}=1.8 \mathrm{~Hz}\right), 4.70\left(p t, 2 \mathrm{H}, J_{\mathrm{H}, \mathrm{H}}=1.8 \mathrm{~Hz}\right) \cdot{ }^{13} \mathrm{C}\left\{{ }^{1} \mathrm{H}\right\}$ NMR $\left(125.7 \mathrm{MHz}, \mathrm{CDCl}_{3}, 298 \mathrm{~K}\right): \delta=55.3\left(\mathrm{C}_{\mathrm{i}}-\mathrm{C}_{5} \mathrm{H}_{4}\right), 72.4$ $\left(\mathrm{C}_{5} \mathrm{H}_{4}\right), 72.9\left(\mathrm{C}_{5} \mathrm{H}_{5}\right), 73.5\left(\mathrm{C}_{5} \mathrm{H}_{4}\right), 119.4(\mathrm{CN})$. HRMS (ESITOF, $M^{+}$): $\mathrm{C}_{11} \mathrm{H}_{9} \mathrm{NRu}: m / z=256.9792$ (calc. 256.9776).

\section{Refinement}

C-bonded $\mathrm{H}$ atoms were placed in calculated positions and constrained to ride on their parent atoms, with $U_{\text {iso }}(\mathrm{H})=$ $1.2 U_{\text {eq }}(\mathrm{C})$ and a $\mathrm{C}-\mathrm{H}$ distance of $0.93 \AA$. Crystal data, data collection and structure refinement details are summarized in Table 2.

\section{Acknowledgements}

MK is grateful to the Fonds der Chemischen Industrie for a Chemiefonds fellowship.

\section{References}

Altmannshofer, S., Herdtweck, E., Köhler, F. H., Miller, R., Mölle, R., Scheidt, E.-W., Scherer, W. \& Train, C. (2008). Chem. Eur. J. 14, 8013-8024.

Barlow, S., Cowley, A., Green, J. C., Brunker, T. J. \& Hascall, T. (2001). Organometallics, 20, 5351-5359.

Bell, W., Ferguson, G. \& Glidewell, C. (1996). Acta Cryst. C52, 19281930.

Bondi, A. (1964). J. Phys. Chem. 68, 441-451.

Bonniard, L., Kahlal, S., Diallo, A. K., Ornelas, C., Roisnel, T., Manca, G., Rodrigues, J., Ruiz, J., Astruc, D. \& Saillard, J. Y. (2011). Inorg. Chem. 50, 114-124.

Borissova, A. O., Antipin, M. Yu., Perekalin, D. S. \& Lyssenko, K. A. (2008). CrystEngComm, 10, 827-832.

Dayaker, G., Sreeshailam, A., Chevallier, F., Roisnel, T., Radha Krishna, P. \& Mongin, F. (2010). Chem. Commun. 46, 2862-2864.

Dowling, N., Henry, P. M., Lewis, N. A. \& Taube, H. (1981). Inorg. Chem. 20, 2345-2348.

Erben, M., Růžička, A., Vinklárek, J., Šť́va, V. \& Handlír, K. (2007). Acta Cryst. E63, m2145-m2146.

Farrugia, L. J. (2012). J. Appl. Cryst. 45, 849-854.

Graham, P. J., Lindsey, R. V., Parshall, G. W., Peterson, M. L. \& Whitman, G. M. (1957). J. Am. Chem. Soc. 79, 3416-3420.

Hildebrandt, A. \& Lang, H. (2013). Organometallics, 32, 5640-5653.

Jakob, A., Ecorchard, P., Köhler, K. \& Lang, H. (2008). J. Organomet. Chem. 693, 3479-3489.

Jakob, A., Ecorchard, P., Linseis, M., Winter, R. F. \& Lang, H. (2009a). J. Organomet. Chem. 694, 655-666.

Jakob, A., Ecorchard, P., Rüffer, T., Linseis, M., Winter, R. F. \& Lang, H. (2009b). J. Organomet. Chem. 694, 3542-3547.

Kivrak, A. \& Zora, M. (2007). J. Organomet. Chem. 692, 2346-2349.

Lang, H., Packheiser, R. \& Walfort, B. (2006). Organometallics, 25, 1836-1850.

Ma, B.-Q. \& Coppens, P. (2003). Chem. Commun. pp. 504-505.

Miesel, D., Hildebrandt, A., Korb, M., Low, P. J. \& Lang, H. (2013). Organometallics, 32, 2993-3002.

Mueller-Westerhoff, U. T., Zheng, Y. \& Ingram, G. (1993). J. Organomet. Chem. 463, 163-167.

Nemykin, V. N., Maximov, A. Y. \& Koposov, A. Y. (2007). Organometallics, 26, 3138-3148.

Oxford Diffraction (2006). CrysAlis CCD and CrysAlis RED. Oxford Diffraction, Abingdon, England.

Packheiser, R., Jakob, A., Ecorchard, P., Walfort, B. \& Lang, H. (2008). Organometallics, 27, 1214-1226.

Poppitz, E. A. A., Hildebrandt, A. M., Korb, M. \& Lang, H. (2014). J. Organomet. Chem. 752, 133-140.

Sato, M., Kawata, Y., Kudo, A., Iwai, A., Saitoh, H. \& Ochiai, S. (1998). J. Chem. Soc. Dalton Trans. pp. 2215-2224.

Sato, M., Kawata, Y., Shintate, H., Habata, Y., Akabori, S. \& Unoura, K. (1997). Organometallics, 16, 1693-1701.

Sato, M., Nagata, T., Tanemura, A., Fujihara, T., Kumakura, S. \& Unoura, K. (2004). Chem. Eur. J. 10, 2166-2178. 
Seiler, P. \& Dunitz, J. D. (1980). Acta Cryst. B36, 2946-2950.

Sheldrick, G. M. (2008). Acta Cryst. A64, 112-122.

Sheldrick, G. M. (2015). Acta Cryst. C71, 3-8.

Sokolov, V. I., Nikitin, L. N., Bulygina, L. A., Khrustalev, V. N., Starikova, Z. A. \& Khokhlov, A. R. (2010). J. Organomet. Chem. 695, 799-803.

Speck, J. M., Claus, R., Hildebrandt, A., Rüffer, T., Erasmus, E., van As, L., Swarts, J. C. \& Lang, H. (2012). Organometallics, 31, 63736380.
Strehler, F., Hildebrandt, H., Korb, M. \& Lang, H. (2013). Z. Anorg. Allg. Chem. 639, 1214-1219.

Strehler, F., Hildebrandt, H., Korb, M., Rüffer, T. \& Lang, H. (2014). Organometallics, 33, 4279-4289.

Westrip, S. P. (2010). J. Appl. Cryst. 43, 920-925.

Wyman, I. W., Robertson, K. N., Cameron, T. S., Swarts, J. C. \& Aquino, M. A. S. (2005). Organometallics, 24, 6055-6058.

Zhang, Y. \& Coppens, P. (2001). Private communication (refcode RALRAX). CCDC, Cambridge, England. 


\section{supporting information}

Acta Cryst. (2015). E71, 398-401 [doi:10.1107/S205698901500540X]

\section{Crystal structure of ruthenocenecarbonitrile}

\section{Frank Strehler, Marcus Korb and Heinrich Lang}

Computing details

Data collection: CrysAlis CCD (Oxford Diffraction, 2006); cell refinement: CrysAlis RED (Oxford Diffraction, 2006); data reduction: CrysAlis RED (Oxford Diffraction, 2006); program(s) used to solve structure: SHELXS97 (Sheldrick, 2008); program(s) used to refine structure: SHELXL2013 (Sheldrick, 2015); molecular graphics: ORTEP-3 for Windows (Farrugia, 2012) and SHELXTL (Sheldrick, 2008); software used to prepare material for publication: WinGX (Farrugia, 2012) and publCIF (Westrip, 2010).

Ruthenocenecarbonitrile

Crystal data

$\left[\mathrm{Ru}\left(\mathrm{C}_{5} \mathrm{H}_{5}\right)\left(\mathrm{C}_{6} \mathrm{H}_{4} \mathrm{~N}\right)\right]$

$M_{r}=256.26$

Monoclinic, $P 2{ }_{1} / m$

$a=7.2023(2) \AA$

$b=8.6802(2) \AA$

$c=7.2922(1) \AA$

$\beta=106.497(2)^{\circ}$

$V=437.12(2) \AA^{3}$

$Z=2$

Data collection

Oxford Gemini S CCD diffractometer

Radiation source: fine-focus sealed tube

Graphite monochromator

$\omega$ scans

Absorption correction: multi-scan

(CrysAlis RED; Oxford Diffraction, 2006)

$T_{\min }=0.849, T_{\max }=1.000$

Refinement

Refinement on $F^{2}$

Least-squares matrix: full

$R\left[F^{2}>2 \sigma\left(F^{2}\right)\right]=0.012$

$w R\left(F^{2}\right)=0.032$

$S=1.05$

900 reflections

67 parameters

0 restraints

Primary atom site location: structure-invariant direct methods
$F(000)=252$

$D_{\mathrm{x}}=1.947 \mathrm{Mg} \mathrm{m}^{-3}$

Mo $K \alpha$ radiation, $\lambda=0.71073 \AA$

Cell parameters from 26762 reflections

$\theta=3.5-28.7^{\circ}$

$\mu=1.74 \mathrm{~mm}^{-1}$

$T=110 \mathrm{~K}$

Block, yellow green

$0.38 \times 0.30 \times 0.30 \mathrm{~mm}$

27710 measured reflections

900 independent reflections

877 reflections with $I>2 \sigma(I)$

$R_{\text {int }}=0.019$

$\theta_{\max }=26.0^{\circ}, \theta_{\min }=3.5^{\circ}$

$h=-8 \rightarrow 8$

$k=-10 \rightarrow 10$

$l=-8 \rightarrow 8$

Secondary atom site location: difference Fourier map

Hydrogen site location: inferred from neighbouring sites

$\mathrm{H}$-atom parameters constrained

$w=1 /\left[\sigma^{2}\left(F_{\mathrm{o}}^{2}\right)+(0.0218 P)^{2}+0.1909 P\right]$

where $P=\left(F_{\mathrm{o}}{ }^{2}+2 F_{\mathrm{c}}{ }^{2}\right) / 3$

$(\Delta / \sigma)_{\max }<0.001$

$\Delta \rho_{\max }=0.27 \mathrm{e} \AA^{-3}$

$\Delta \rho_{\min }=-0.39$ e $\AA^{-3}$ 


\section{Special details}

Geometry. All e.s.d.'s (except the e.s.d. in the dihedral angle between two 1.s. planes) are estimated using the full covariance matrix. The cell e.s.d.'s are taken into account individually in the estimation of e.s.d.'s in distances, angles and torsion angles; correlations between e.s.d.'s in cell parameters are only used when they are defined by crystal symmetry. An approximate (isotropic) treatment of cell e.s.d.'s is used for estimating e.s.d.'s involving 1.s. planes.

Refinement. Refinement of $F^{2}$ against ALL reflections. The weighted $R$ factor $w R$ and goodness of fit $S$ are based on $F^{2}$, conventional $R$ factors $R$ are based on $F$, with $F$ set to zero for negative $F^{2}$. The threshold expression of $F^{2}>\sigma\left(F^{2}\right)$ is used only for calculating $R$ factors(gt) etc. and is not relevant to the choice of reflections for refinement. $R$ factors based on $F^{2}$ are statistically about twice as large as those based on $F$, and $R$ factors based on ALL data will be even larger.

Fractional atomic coordinates and isotropic or equivalent isotropic displacement parameters $\left(\hat{A}^{2}\right)$

\begin{tabular}{lllll}
\hline & $x$ & $y$ & $z$ & $U_{\text {iso }} * / U_{\text {eq }}$ \\
\hline C1 & $-0.4152(3)$ & 0.2500 & $-0.0364(3)$ & $0.0162(4)$ \\
C2 & $-0.3084(3)$ & 0.2500 & $0.1617(3)$ & $0.0142(4)$ \\
C3 & $-0.24721(18)$ & $0.11497(16)$ & $0.27791(19)$ & $0.0142(3)$ \\
H3C & -0.2679 & 0.0130 & 0.2382 & $0.017^{*}$ \\
C4 & $-0.14854(18)$ & $0.16776(15)$ & $0.46603(18)$ & $0.0145(3)$ \\
H4C & -0.0935 & 0.1053 & 0.5710 & $0.017^{*}$ \\
C5 & $0.1429(3)$ & 0.2500 & $0.0334(3)$ & $0.0185(4)$ \\
H5C & 0.0744 & 0.2500 & -0.0957 & $0.022^{*}$ \\
C6 & $0.20428(19)$ & $0.11674(17)$ & $0.1491(2)$ & $0.0175(3)$ \\
H6C & 0.1832 & 0.0149 & 0.1089 & $0.021^{*}$ \\
C7 & $0.30392(17)$ & $0.16746(16)$ & $0.33757(19)$ & $0.0158(3)$ \\
H7C & 0.3591 & 0.1045 & 0.4420 & $0.019^{*}$ \\
N1 & $-0.5024(2)$ & 0.2500 & $-0.1949(3)$ & $0.0244(4)$ \\
Ru1 & $0.00474(2)$ & 0.2500 & $0.26311(2)$ & $0.00953(7)$ \\
& & &
\end{tabular}

Atomic displacement parameters $\left(\AA^{2}\right)$

\begin{tabular}{lllllll}
\hline & $U^{11}$ & $U^{22}$ & $U^{33}$ & $U^{12}$ & $U^{13}$ & $U^{23}$ \\
\hline $\mathrm{C} 1$ & $0.0120(8)$ & $0.0168(9)$ & $0.0198(10)$ & 0.000 & $0.0045(7)$ & 0.000 \\
$\mathrm{C} 2$ & $0.0093(8)$ & $0.0168(9)$ & $0.0170(9)$ & 0.000 & $0.0044(7)$ & 0.000 \\
$\mathrm{C} 3$ & $0.0111(6)$ & $0.0148(7)$ & $0.0182(6)$ & $-0.0022(5)$ & $0.0065(5)$ & $-0.0003(5)$ \\
$\mathrm{C} 4$ & $0.0145(6)$ & $0.0163(7)$ & $0.0146(6)$ & $0.0003(5)$ & $0.0072(5)$ & $0.0029(5)$ \\
$\mathrm{C} 5$ & $0.0155(9)$ & $0.0283(11)$ & $0.0145(9)$ & 0.000 & $0.0087(7)$ & 0.000 \\
$\mathrm{C} 6$ & $0.0143(6)$ & $0.0190(7)$ & $0.0222(7)$ & $0.0005(5)$ & $0.0101(5)$ & $-0.0047(6)$ \\
$\mathrm{C} 7$ & $0.0098(6)$ & $0.0191(7)$ & $0.0193(6)$ & $0.0033(5)$ & $0.0054(5)$ & $0.0029(5)$ \\
$\mathrm{N} 1$ & $0.0222(9)$ & $0.0274(10)$ & $0.0208(9)$ & 0.000 & $0.0015(7)$ & 0.000 \\
Ru1 & $0.00850(10)$ & $0.00992(10)$ & $0.01040(10)$ & 0.000 & $0.00305(6)$ & 0.000 \\
\hline
\end{tabular}

Geometric parameters $\left(\AA,^{\circ}\right)$

\begin{tabular}{llll}
\hline $\mathrm{C} 1-\mathrm{N} 1$ & $1.148(3)$ & $\mathrm{C} 5-\mathrm{Ru} 1$ & $2.1780(18)$ \\
$\mathrm{C} 1-\mathrm{C} 2$ & $1.431(3)$ & $\mathrm{C} 5-\mathrm{H} 5 \mathrm{C}$ & 0.9300 \\
$\mathrm{C} 2-\mathrm{C} 3^{\mathrm{i}}$ & $1.4401(17)$ & $\mathrm{C} 6-\mathrm{C} 7$ & $1.4274(19)$ \\
$\mathrm{C} 2-\mathrm{C} 3$ & $1.4401(17)$ & $\mathrm{C} 6-\mathrm{Ru} 1$ & $2.1848(13)$ \\
$\mathrm{C} 2-\mathrm{Ru} 1$ & $2.1649(18)$ & $\mathrm{C} 6-\mathrm{H} 6 \mathrm{C}$ & 0.9300 \\
$\mathrm{C} 3-\mathrm{C} 4$ & $1.4294(18)$ & $\mathrm{C} 7-7^{\mathrm{i}}$ & $1.433(3)$
\end{tabular}




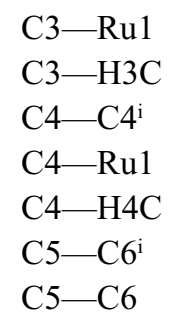

$2.1885(13)$

0.9300

$1.428(3)$

2.2013 (12)

0.9300

$1.4262(18)$

$1.4262(18)$

179.4 (2)

$125.52(8)$

$125.52(8)$

$108.96(16)$

123.64 (13)

71.57 (8)

71.57 (8)

$106.82(12)$

$71.48(7)$

$69.80(9)$

126.6

126.6

123.8

$108.70(8)$

71.08 (3)

70.51 (7)

125.7

125.7

124.4

108.40 (17)

71.18 (9)

71.18 (9)

125.8

125.8

123.5

$107.83(13)$

$70.66(9)$

71.06 (7)

126.1

126.1

123.8

107.97 (8)

$70.83(7)$

70.88 (4)

126.0

126.0

123.9

$113.36(7)$

$127.17(5)$

$38.16(5)$
C7- Ru 1

C7-H7C

$\mathrm{Ru} 1-\mathrm{C}^{\mathrm{i}}$

$\mathrm{Ru} 1-\mathrm{C}^{\mathrm{i}}$

$\mathrm{Ru} 1-\mathrm{C}^{\mathrm{i}}$

$\mathrm{Ru} 1-\mathrm{C} 4^{\mathrm{i}}$

$2.1878(12)$

0.9300

2.1848 (13)

$2.1878(12)$

2.1885 (13)

2.2013 (12)

C5-Ru1-C6

$38.16(5)$

C6 - Ru1-C6

$\mathrm{C} 2-\mathrm{Ru} 1-\mathrm{C}^{\mathrm{i}}$

$\mathrm{C} 5-\mathrm{Ru} 1-\mathrm{C}^{\mathrm{i}}$

$\mathrm{C} 6$ - ${ }^{\mathrm{R} u} 1-\mathrm{C} 7^{\mathrm{i}}$

$\mathrm{C} 6-\mathrm{Ru} 1-\mathrm{C}^{\mathrm{i}}$

$\mathrm{C} 2-\mathrm{Ru} 1-\mathrm{C} 7$

C5-Ru1-C7

C6 $-\mathrm{Ru} 1-\mathrm{C} 7$

C6-Ru1-C7

$\mathrm{C} 77^{\mathrm{i}}-\mathrm{Ru} 1-\mathrm{C} 7$

$\mathrm{C} 2-\mathrm{Ru} 1-\mathrm{C}^{\mathrm{i}}$

$\mathrm{C} 5-\mathrm{Ru} 1-\mathrm{C}^{\mathrm{i}}$

$\mathrm{C} 6{ }^{\mathrm{i}}-\mathrm{Ru} 1-\mathrm{C} 3^{\mathrm{i}}$

C6-Ru1-C3 ${ }^{\mathrm{i}}$

$\mathrm{C} 7{ }^{\mathrm{i}}-\mathrm{Ru} 1-\mathrm{C}^{\mathrm{i}}$

$\mathrm{C} 7-\mathrm{Ru} 1-\mathrm{C} 3^{\mathrm{i}}$

$\mathrm{C} 2-\mathrm{Ru} 1-\mathrm{C} 3$

$\mathrm{C} 5-\mathrm{Ru} 1-\mathrm{C} 3$

$\mathrm{C} 6$ - $\mathrm{Ru} 1-\mathrm{C} 3$

C6-Ru1-C3

$\mathrm{C} 7 \mathrm{i}-\mathrm{Ru} 1-\mathrm{C} 3$

C7-Ru1-C3

C3 $3^{\mathrm{i}-\mathrm{Ru} 1-\mathrm{C} 3}$

$\mathrm{C} 2-\mathrm{Ru} 1-\mathrm{C} 4^{\mathrm{i}}$

$\mathrm{C} 5-\mathrm{Ru} 1-\mathrm{C} 4^{\mathrm{i}}$

$\mathrm{C} 6$ - $\mathrm{Ru} 1-\mathrm{C} 4$

C6- $\mathrm{Ru} 1-\mathrm{C} 4^{\mathrm{i}}$

$\mathrm{C} 7{ }^{\mathrm{i}}-\mathrm{Ru} 1-\mathrm{C}_{4}^{\mathrm{i}}$

$\mathrm{C} 7-\mathrm{Ru} 1-\mathrm{C} 4^{\mathrm{i}}$

$\mathrm{C} 3^{\mathrm{i}}-\mathrm{Ru} 1-\mathrm{C} 4^{\mathrm{i}}$

$\mathrm{C} 3-\mathrm{Ru} 1-\mathrm{C} 4^{\mathrm{i}}$

$\mathrm{C} 2-\mathrm{Ru} 1-\mathrm{C} 4$

C5-Ru1-C4

C6 $-\mathrm{Ru} 1-\mathrm{C} 4$

C6-Ru1-C4

$\mathrm{C} 7{ }^{\mathrm{i}}-\mathrm{Ru} 1-\mathrm{C} 4$

C7-Ru1-C4

$\mathrm{C} 3$ - $\mathrm{Ru} 1-\mathrm{C} 4$

$\mathrm{C} 3-\mathrm{Ru} 1-\mathrm{C} 4$
$63.94(8)$

160.38 (4)

63.77 (6)

$38.11(5)$

$63.89(5)$

160.38 (4)

63.77 (6)

$63.89(5)$

38.11 (5)

38.23 (7)

38.63 (4)

$127.17(5)$

112.30 (6)

$161.19(5)$

$125.76(5)$

$159.25(5)$

38.63 (4)

$127.17(5)$

161.18 (5)

$112.30(6)$

$159.25(5)$

$125.76(5)$

$64.76(7)$

$63.69(6)$

160.55 (4)

126.43 (5)

$159.58(5)$

$111.94(5)$

$125.87(5)$

$38.01(5)$

$63.86(5)$

63.69 (6)

160.55 (4)

$159.58(5)$

$126.42(5)$

125.87 (5)

111.94 (5)

$63.86(5)$

38.01 (5) 
C2-Ru1-C6

$\mathrm{C} 1-\mathrm{C} 2-\mathrm{C} 3-\mathrm{C} 4$

$\mathrm{C} 3-\mathrm{C} 2-\mathrm{C} 3-\mathrm{C} 4$

$\mathrm{Ru} 1-\mathrm{C} 2-\mathrm{C} 3-\mathrm{C} 4$

$\mathrm{C} 1-\mathrm{C} 2-\mathrm{C} 3-\mathrm{Ru} 1$

$\mathrm{C} 3-\mathrm{C} 2-\mathrm{C} 3-\mathrm{Ru} 1$

$\mathrm{C} 2-\mathrm{C} 3-\mathrm{C} 4-\mathrm{C}^{\mathrm{i}}$

$\mathrm{Ru} 1-\mathrm{C} 3-\mathrm{C} 4-\mathrm{C} 4^{\mathrm{i}}$
$127.17(5)$

$-179.04(16)$

$0.13(19)$

$62.30(9)$

$118.66(18)$

$-62.17(12)$

$-0.08(12)$

$61.12(4)$
$\mathrm{C} 4 \mathrm{i}-\mathrm{Ru} 1-\mathrm{C} 4$

$\mathrm{C} 2-\mathrm{C} 3-\mathrm{C} 4-\mathrm{Ru} 1$

$\mathrm{C} 6-\mathrm{C} 5-\mathrm{C} 6-\mathrm{C} 7$

$\mathrm{Ru} 1-\mathrm{C} 5-\mathrm{C} 6-\mathrm{C} 7$

$\mathrm{C} 6-\mathrm{C} 5-\mathrm{C} 6-\mathrm{Ru} 1$

$\mathrm{C} 5-\mathrm{C} 6-\mathrm{C} 7-\mathrm{C}^{\mathrm{i}}$

$\mathrm{Ru} 1-\mathrm{C} 6-\mathrm{C} 7-\mathrm{C}^{\mathrm{i}}$

$\mathrm{C} 5-\mathrm{C} 6-\mathrm{C} 7-\mathrm{Ru} 1$
$37.84(7)$

$-61.20(10)$

$0.1(2)$

$-61.69(9)$

$61.79(12)$

$-0.06(12)$

$-61.50(4)$

$61.44(10)$

Symmetry code: (i) $x,-y+1 / 2, z$. 